\title{
Suppressing of Irreversible Capacity in Lithium-ion Batteries
}

\author{
Jiri Libich, Jiri Vondrák, Marie Sedlařiková, Josef Máca, Martin Frk \\ Department of Electrical and Electronic Technology, The Faculty of Electrical Engineering and \\ Communication, Brno University of Technology, Brno Technická 3058/10 616 00, Czech Republic
}

Received 1 July 2013; accepted 23 December 2013

\begin{abstract}
The article deals with preparing of lithiated graphite material. The lithiated graphite material can be used as active electrode material in lithium-ion batteries. Most of the commercially produced lithium-ion batteries have the negative electrode based on graphite. The capacity losses which are caused by irreversible capacity of graphite may reduce the potential capacity of the battery from $15 \%$ up to $45 \%$. These losses arise on negative electrode interphase (solid graphite electrode and liquid electrolyte), where during the formation process is created the SEI (Solid-Electrolyte Interphase) layer. The layer is composed from lithium atoms and the decomposition products of electrolyte solvents. The SEI layer is indispensable for correct function of lithium-ion battery. In this article are presented experimental methods for synthesis of lithiated graphite material. The article describes the three concepts of preparing lithiated material and its using like a precursor for preparing of negative electrode. The first method is based on using n-butyllithium $\left(\mathrm{C}_{4} \mathrm{H}_{9} \mathrm{Li}\right)$ reagent, its behaviour as the donor of lithium atoms, and graphite acts as the acceptor of lithium atoms. The second concept follows the first one only with adding an ionic compound to graphite, in our case $\mathrm{FeCl}_{3}$. The last concept presents the electroless lithiation process, which is based on different electrochemical potential between lithium and graphite.
\end{abstract}

Keywords: lithium, graphite, irreversible capacity, lithiation process.

\section{Introduction}

The capacity losses, which are caused by irreversible capacity of graphite, arise on negative electrode between the first and second charge-discharge cycles. During the first charging two redox reactions occur among lithium cations $\left(\mathrm{Li}^{+}\right)$ and molecules of solvent. These reactions proceed on the electrode surface; the magnitude of the losses depends on the specific surface area of the graphite material. These reactions lead to origin of SEI layer. The layer is comprised from the products of redox reactions, for example $\mathrm{Li}_{2} \mathrm{CO}_{3}, \mathrm{LiO}_{2}, \mathrm{C}_{2} \mathrm{H}_{4}, \mathrm{CO}_{3}{ }^{2-}$ and the

\footnotetext{
* Corresponding author. E-mail address: xlibic00@stud.feec.vutbr.cz
} 
other inorganic and organic compounds. The exact composition of SEI layer depends on the kind of solvents which are used, being the widely used a mixture of dimethyl carbonate (DMC) and ethylene carbonate (EC). In our experiments we have used the mixture of DMC and EC in a ratio of $50 \mathrm{wt} \%$ to $50 \mathrm{wt} \%$. SEI layer has got a specific conductive property: it works like a membrane that is transparent for lithium atoms but for electrons is nonconductive. This characteristic property of SEI layer provides to lithium-ion batteries very low self-discharge rate.

In our research we have used two types of crystalline graphite: natural and expanded graphite (see main properties in Table 1). These two graphite types represent the most widely used materials for negative electrode in lithium-ion batteries. Our idea is to prepare a graphite electrode that already contains lithium atoms before the first charging cycle. These lithium atoms can be used and consumed to SEI layer creation. The lithium atoms which are in graphite are used in the first and second charge-discharge cycles. The entire capacity of positive electrode is preserved and its lithium atoms can be used for charge transfer $[2,8,9,11,12]$.

Table 1. The basic parameters of graphite materials.

\begin{tabular}{|c|c|c|c|}
\hline Type & Particle diameter $[\mu \mathrm{m}]$ & Specific surface $\left[\mathrm{m}^{2} \cdot \mathrm{g}^{-1}\right]$ & Sheet distances $[\mathrm{nm}]$ \\
\hline Natural & $<11$ & 10 & 0.336 \\
\hline Expanded & $<150$ & 68 & 1.2 \\
\hline
\end{tabular}

\section{Experimental setup}

Our work is divided into three parts which describe three different lithiation methods. The first part presents the experiment process, where n-butyllithium reagent as donor of lithium atoms is used. The measurements showed, despite our hypothesis, that n-butyllithium is now able to reduce graphite material, i.e., inserting lithium atoms among graphite sheets. The second part of our research is based on the results obtained in the first part. In this experiment we have used the ionic compound $\mathrm{FeCl}_{3}$ as lithium attractor. Along with our assumption, the ionic molecule had tear off the lithium atom from butyl chain. The separated lithium atom should be easier inserted or bonded to the graphite structure. The third part performs a special lithiation process that is based on conductive contention between the graphite electrode and metal lithium. If the graphite electrode is dipped in an aprotic electrolyte together with the metal lithium, we get a half-cell with an electrochemical potential of approximately $2.5 \mathrm{~V}$. This way, the prepared half-cell can be connected via outer conductor; if this is done, the electric current begins to flow through the conductor. Based on the electrochemical potential of the cell, the lithium cations are transported through the electrolyte to graphite electrode where they are reduced from outer circuit. In this case, we call it electroless lithiation process $[1,5,3,7]$. 


\section{Manufacturing process}

This section describes the steps of manufacturing process which were used to preparing lithiated graphite. The manufacturing process is focused on doping graphite by lithium ions. The lithiated graphite material served as precursor for preparing the final graphite electrode. All steps were carried out under inert atmosphere of argon. The n-butyllithium reagent used in our experiments contains 2.5 molar concentration of n-butyllithium dissolved in n-hexane. An electrolyte containing 1 molar concentration of $\mathrm{LiPF}_{6}$ salt was used in all the experiments $[13,14]$.

\section{A. Lithiation with help of n-butyllithium}

1. Graphite material was dried at a temperature of $150{ }^{\circ} \mathrm{C}$ for 12 hours in a vacuum chamber. This step is necessary to remove the residual moisture from graphite. The n-butyllithium is very reactive with water.

2. Addition of the n-butyllithium reagent into the graphite. The molar ratio was calculated: one $\mathrm{Li}$ atom per six carbon atoms $-\mathrm{LiC}_{6}$.

3. The mixture of n-butyllithium and graphite was stirred for 24 hours under room temperature.

4. Following up the prior stirring step, the mixture was filtered. The lithiated graphite blend was purged using n-hexane solvent in order to remove its $n$ butyllithium residues. The mixture is stirred with n-hexane for 6 hours.

5. After the purging step, the mixture was a second time filtered. Now, the lithiated graphite is in its final state (precursor for preparing the testing electrode) and it should not contain any residues.

B. Lithiation with help of n-butyllithium and iron(III) chloride $\left(\mathrm{FeCl}_{3}\right)$ attractor

1. Graphite material was dried at a temperature of $150{ }^{\circ} \mathrm{C}$ for 12 hours in a vacuum chamber.

2. The $\mathrm{FeCl}_{3}$ compound has been dried in a vacuum chamber at a temperature of $150{ }^{\circ} \mathrm{C}$ for 24 hours. It is very important to remove the residual moisture from $\mathrm{FeCl}_{3}$ powder.

3. Both dried components (graphite and $\mathrm{FeCl}_{3}$ ) were mixed together in an exact ratio. The ratios were calculated as follows: one hexagonal unit $\left(\mathrm{C}_{6}\right)$ of graphite per one $\mathrm{FeCl}_{3}$ molecule. The two ratios of the components were prepared as follows:

- first ratio, ten carbon atoms per one $\mathrm{FeCl}_{3}$ molecule, ratio 10:1.

- second ratio, six carbon atoms per one $\mathrm{FeCl}_{3}$ molecule, ratio 6:1.

Each composition ratio was stirred with n-hexane for 24 hours, this process making the composition more homogeneous.

4. The mixture that contains the exact ratio of graphite/ $\mathrm{FeCl}_{3}$ and $\mathrm{n}$-hexane was filtered and dried in order to remove n-hexane solvent.

5. Addition of the n-butyllithium reagent into the mixture. The molar ratio was calculated: one $\mathrm{Li}$ atom per six carbon atoms $-\mathrm{LiC}_{6}$. The following process steps are the same as in paragraph $A$, point 3 (above). 


\section{Electroless lithiation process}

1. Graphite material was dried at a temperature of $150{ }^{\circ} \mathrm{C}$ for 12 hours in a vacuum chamber.

2. The negative electrode was prepared from the dried graphite material. For improving of material properties, $10 \mathrm{wt} \%$ of binder (polyvinylidenefluoride PVDF) were added to the graphite material.

3. Electrode material was coated onto copper foil (thickness $35 \mu \mathrm{m}$ ) and was sintered at the temperature of $50{ }^{\circ} \mathrm{C}$ for 24 hours.

4. The prepared electrode was connected to the system that is shown in Fig. 7. During this step, the electrode material was reduced by lithium ions for 48 hours, from the potential of $2.5 \mathrm{~V}$ up to $100 \mathrm{mV}$.

5. In the last stage of this process, the electrode material was stripped off from the copper collector and dried out under room temperature. This lithiated material was used like a precursor for preparing the testing electrode.

\section{Experimental results}

In our experiments we have used two types of graphite: expanded graphite (manufacturer Bochemie, a.s.) and natural graphite - COND CR5995 (manufacturer Graphite Tyn.). The main parameters of each graphite type are listed in Erro! A origem da referência não foi encontrada..

\section{Graphite lithiation with help of n-butyllithium}

This section deals with preparing negative electrodes from prelithiated graphite. These electrodes were made for experimental and measurement purposes. The manufacturing process begins from the precursor (lithiated graphite material), which was obtained by lithiation process, as quoted above.

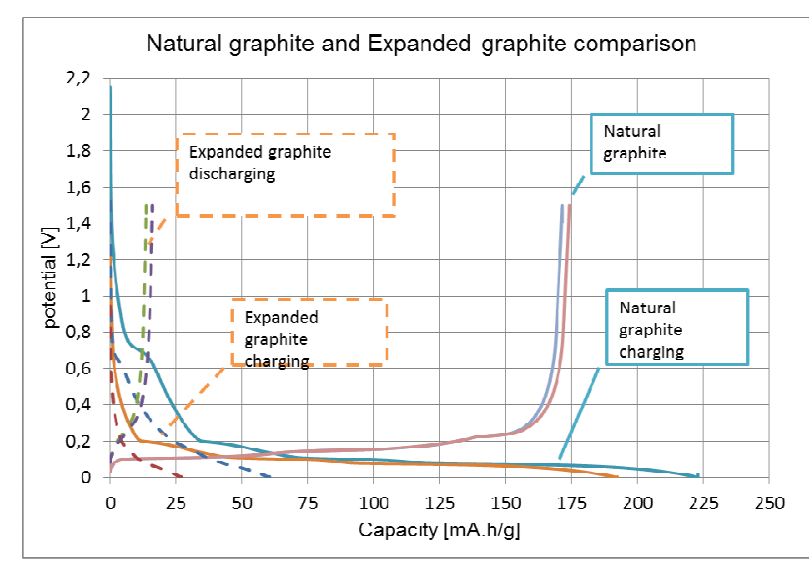

Figure 1. Charge-discharge capacity characteristics.

Through the manufacturing steps, we have got the lithiated graphite material in powder form. The lithiated graphite powder was mixed with PVDF binder. The binder was calculated as 10wt\% of lithiated graphite material and must be dissolved in N-methyl-2-pyrrolidone (NMP) solvent. The electrode mixture was stirred for 24 hours. After stirring the blend (mixture) was coated on a copper foil (thickness $35 \mu \mathrm{m}$ ). The wet thickness of the coated layer was $80 \mu \mathrm{m}$; in the next 
step the coated blend was sintered for 12 hours at the temperature of $50{ }^{\circ} \mathrm{C}$. As final step of the electrode manufacturing process small rounded electrodes with $18 \mathrm{~mm}$ diameter were cut off. These electrodes were pressed by a pressure of 2 tons per $\mathrm{cm}^{2}$. The electrodes were subsequently measured on a potentiostat /galvanostat instrument in a half-cell system. Arrangement of the electrodes was as follows: working electrode (WE) connecting to analysed electrode, counter electrode (CE) was represented by metal lithium. At the beginning of the two types of graphite were measured and compared, before being used in our experiments (see Fig. 1). The graph displayed in Fig. 2 shows measured characteristics of expanded graphite. The graph compares the characteristics of lithiated and non-lithiated material. The electrode which was made from nonlithiated material (dashed curve) has got about $20 \%$ higher capacity than the lithiated one (solid line).

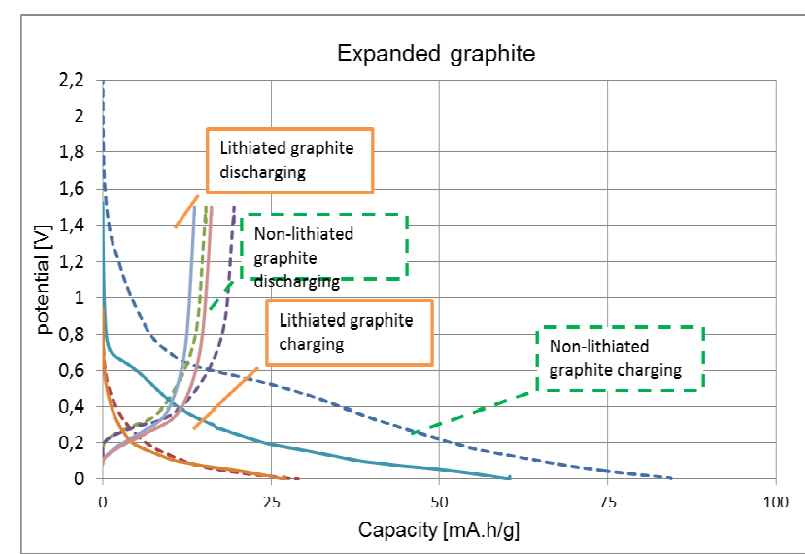

Figure 2. Charge-discharge characteristics of expanded graphite.

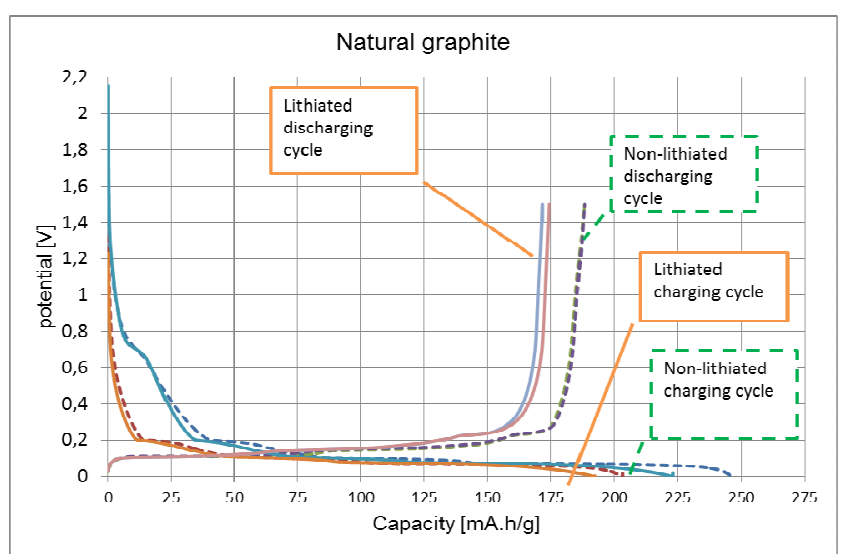

Figure 3. Charge-discharge characteristics of natural graphite.

The graph presented in Fig. 3 shows the measured characteristics of natural graphite. The electrode which was made from non-lithiated material (dashed curve) has higher capacity than the lithiated one (solid line), as in the previous case. Natural graphite has not got the difference of capacity as big as in the case of the expanded graphite $[1,4]$.

The both types of graphite electrodes (lithiated and non-lithiated) have got approximately similar irreversible capacity. The non-lithiated natural graphite material has an irreversible capacity of around the $24 \%$, and the lithiated one has 
an irreversible capacity of around $23 \%$, see Fig. 3. The more noticeable difference can be seen in the case of expanded graphite, Fig. 2. The non-lithiated electrode material has an irreversible capacity of around $82 \%$, and the lithiated electrode material has around $77 \%$. The summarized results are listed in Table 2.

Table 2. Properties of lithiated graphite materials.

\begin{tabular}{|c|c|c|c|c|}
\hline \multirow{2}{*}{ Type } & \multicolumn{2}{|c|}{ Stabilized capacity [mA.h/g] } & \multicolumn{2}{c|}{ Irreversible capacity [\%] } \\
\cline { 2 - 5 } & Lithi. & Non Lithi. & Lithi. & Non Lithi. \\
\hline Natural & 188 & 203 & 23 & 24 \\
\hline Expanded & 27 & 28 & 77 & 82 \\
\hline
\end{tabular}

Lithi. means lithiated graphite material; Non Lithi. means initial graphite material without any litihum atoms.

\section{Graphite doped with iron(III) chloride $\left(\mathrm{FeCl}_{3}\right)$}

The experiments presented in this section are based on the results obtained in the previous section (Graphite lithiation with help of n-butyllithium). In Table 2 it is clearly seen the poor capacity characteristics of expanded graphite; the following experiments were carried out with natural graphite CR 5995 provided by Graphite Tyn company. In terms of irreversible capacity, only a slight difference between lithiated and non-lithiated graphite material was observed. There is a missing intercalation reaction between n-butyllithium reagent and the graphite material.

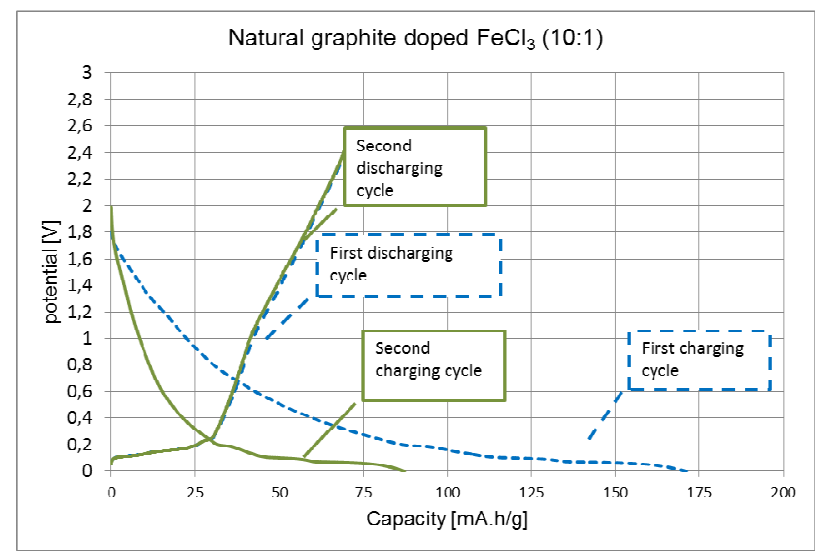

Figure 4. Charge-discharge characteristics of graphite doped by $\mathrm{FeCl}_{3}$ in the ratio 10:1.

In the other experiments the ionic compound iron(III) chloride $\left(\mathrm{FeCl}_{3}\right)$ was used. $\mathrm{FeCl}_{3}$ belongs to halides group. Halides are binary compounds, of which one part is a halogen atom and the other part is an element which is more electropositive. The halide group contains the significant compounds copper(II) chloride $\left(\mathrm{CuCl}_{2}\right)$, manganese(II) chloride $\left(\mathrm{MnCl}_{2}\right)$ and cobalt(II) chloride $\left(\mathrm{CoCl}_{2}\right)$. All of these compounds are suitable for using in a lithiation process. We have decided to use $\mathrm{FeCl}_{3}$ due to its three chloride atoms which cause partial distribution of the charge $[3,4]$. The manufacturing process of the electrode material has been 
previously described. It was coated on a copper foil with the addition of a binder. The manufacturing process of the electrodes is the same as described in previous the case. The electrodes were subsequently measured on a potentiostat /galvanostat instrument (Bio-Logic) in a half-cell system. The measured characteristics are displayed in Fig. 4 and Fig 5.

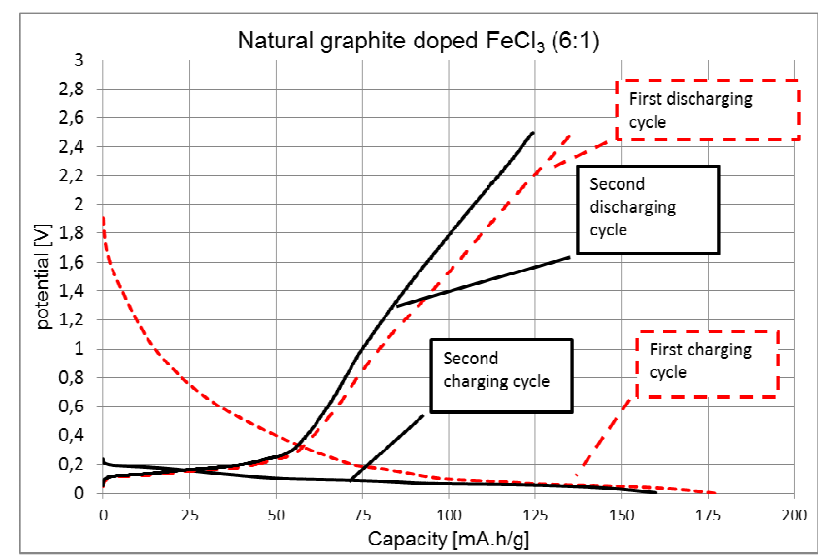

Figure 5. Charge-discharge characteristics of graphite doped by $\mathrm{FeCl}_{3}$ in the ratio 6:1.

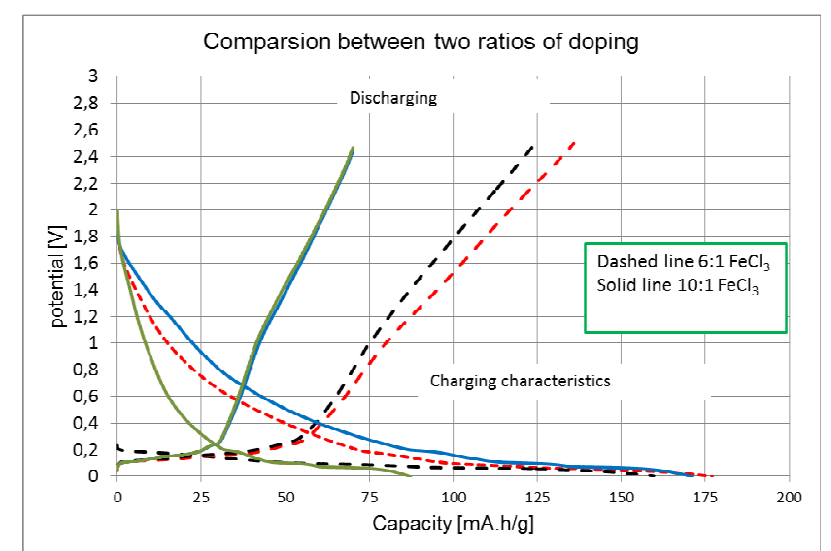

Figure 6. Comparison between two ratios of $\mathrm{FeCl}_{3}$.

The obtained results show the capacity characteristics of the measured specimens. The electrode material with the ratio 10:1 (ten carbon atoms per one atom of $\mathrm{FeCl}_{3}$ ) has lower stable capacity (around $85 \mathrm{~mA} . \mathrm{h} / \mathrm{g}$ ) and higher irreversible capacity (58\%) than the electrode material with the ratio $6: 1$, see Fig. 4. The material with the ratio $6: 1$ has a stable capacity of around 160 $\mathrm{mA} . \mathrm{h} / \mathrm{g}$, and an irreversible capacity of $15 \%$, see Fig. 4. This marked difference of the material characteristics is evident from Fig. 6 and Table 3.

The differences of capacity characteristics are displayed in Fig. 6. Measured results of that experiment are given in Table 3 and Table 4.

Table 3. Obtained values of irreversible and steady capacity.

\begin{tabular}{|c|c|c|}
\hline Ratio $\mathrm{FeCl}_{3}$ (natural) & Stabilized capacity [mA.h/g] & Irreversible capacity [\%] \\
\hline $10: 1$ & 87 & 58 \\
\hline $6: 1$ & 162 & 15 \\
\hline
\end{tabular}


Table 4. Conclusion summary.

\begin{tabular}{|c|c|c|}
\hline Type & Stable capacity [mA.h/g] & Irreversible capacity [\%] \\
\hline Pure & 203 & 24 \\
\hline Lithiated & 188 & 23 \\
\hline Doped $\mathrm{FeCl}_{3}(10: 1)^{2}$ & 87 & 58 \\
\hline Doped $\mathrm{FeCl}_{3}(6: 1)$ & 162 & 15 \\
\hline Electroless & 210 & 2 \\
\hline
\end{tabular}

Pure, means initial graphite material (COND CR5995), without any modification. Lithiated, means natural graphite lithiated with help of $2.5 \mathrm{M}$ n-butyllithium. Doped, means natural graphite doped by $\mathrm{FeCl}_{3}$ attractor and lithiated with help of $2.5 \mathrm{M} \mathrm{n}$ - butyllithium.

\section{Electroless lithiation process}

The previous experiments give only a small improving of the irreversible capacity as well as of the material characteristics. There is only negligible improvement of irreversible capacity along with steady material capacity. This third section deals with the simple lithiation method that is based on the potential of the electrochemical cell. The electrochemical potential is formed between the graphite electrode (material based on graphite with additives that is plated on the copper collector) and the electrode from metal lithium $[6,7,10]$. This half-cell is connected through a wire that serves as outer conductor of electron (see Fig. 7).

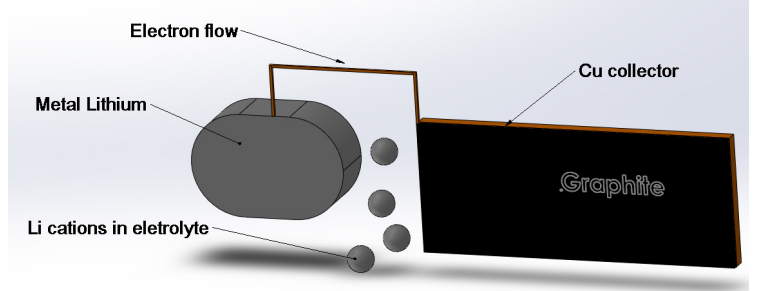

Figure 7. Conception of electroless lithiation system.

The system presented in Fig. 7 was used in our process of electrode material manufacturing. As an electrolyte it was used $1 \mathrm{M}$ concentration of $\mathrm{LiClO}_{4}$ salt (dissolved in a mixture of DMC and EC, as described above). This way, the prepared material was coated by the electrode collector, following the steps described formerly. Capacity characteristics obtained from pre-lithiated graphite are shown in Fig. 8. 


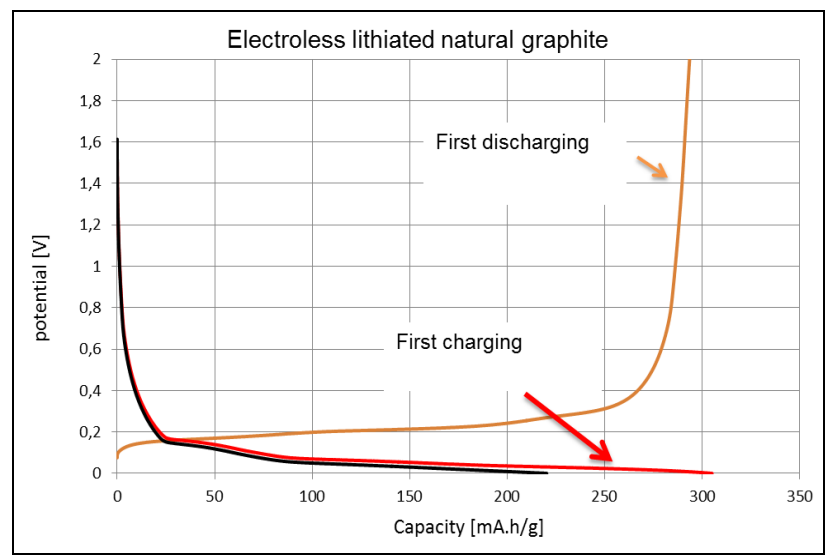

Figure 8. Capacity characteristics of pre-lithiated natural graphite (COND CR 5995).

The values of irreversible and steady capacity, measured with the help of the electroless lithiation process, brought significant improvement. This lithiation process fully saturated the graphite structure by lithium atoms. These atoms are used during the electrode formation (they are consumed in the development of the SEI layer on the heterogeneous interphase electrode/electrolyte); also, they prepare the positions inside the graphite structure for the other lithium atoms in the subsequent charge-discharge cycles.

\section{Conclusions}

In the first part of our experimental work (Graphite lithiation with help of $n$ butyllithium) we describe the lithiation process with the help of $n$ - butyllithium reagent. In the experiments two graphite kinds were used: expanded and natural (CR5995) graphite, see Tabel 1. Fig. 2 and Fig. 3 show the comparison between lithiated and non-lithiated materials. The expanded graphite shows decrease of capacity which can be caused by the sheet distances extension occurred during the lithiation process. The expanded graphite was taken out from other experiments due to its small and high irreversible capacity, see Fig. 1. The natural graphite does not show this marked difference at capacity characteristics. The irreversible capacity of natural graphite was slightly improved from $24 \%$ to $23 \%$. The $n$ - butyllithium is a too feeble reduction agent for graphite. The $\mathrm{Li}^{+}$ atoms are not able to separate from n-butyl chain and intercalating to graphite.

The second part of our work (Graphite doped by iron(III) chloride $\left(\mathrm{FeCl}_{3}\right)$ ) uses the iron(III) chloride ionic compound like a lithium attractor. Obtained results are listed in Table 3. The most suitable ratio of iron(III) chloride is $1: 6$, i.e., one molecule of $\mathrm{FeCl}_{3}$ per six carbon atoms (similar as non-stoichiometric compound $\mathrm{LiC}_{6}$ ). This experiment with ionic compound got decreasing of irreversible capacity from $24 \%$ to $15 \%$.

The last part of our work (Electroless lithiation process) brought the expected characteristics, see Fig. 8. The irreversible capacity of natural graphite decreased from $24 \%$ to $2 \%$.

The overall results are summarized and compared in Table 4. Our experiments confirm that the idea of irreversible capacity decreasing by using pre-lithiated graphite material works. The electroless lithiation method for preparing pre- 
lithiated electrode material is theme to discussion. Its utilization and application in commercial area can be difficult but it can bring higher capacities and better characteristic of lithium-ion batteries.

\section{Acknowledgments}

This work was supported by the project Support for human resources and transfer of knowledge in conditions of international cooperation of research teams CZ.1.07/2.3.00/20.0103, Grant Agency of Czech Republic, Project P102/10/2091 (Increase the safety of lithium-ion batteries) and specific research FEKT-S-11-7 (Materials and technologies for electronics).

\section{References}

1. Yazami R. Synthetic Met. 1987;20:383-386.

2. Zhao L, Watanabe I, Doi T, et al. J Power Sources. 2006;161:1275-1280.

3. Dines MB. Mater Res Bull. 1975;10:287-291.

4. Yazami R. Synthetic Met. 1983;7:169-176.

5. Hung CC. Carbon. 1995;33:315-322.

6. Ratnakumar BV, Smart MC, Surampudi S. J Power Sources. 2001;9798:137-139

7. $\quad$ Sang-Pil K, Adri CT, Van D. et al. J Power Sources. 2011;196:8590-8597.

8. Smart MC, Ratnakumar BV, Surampudi S, et al. J Electrochem Soc. 1999;146:3963-3969.

9. Sivakkumar SR, Pandolfo AG. Electrochim Acta. 2012;65:280-287.

10. Holzapfel M, Martinent A. J Electroanal Chem. 2003;546:41-50

11. Huang S, Fan F, Li J, et al. Acta Mater. 2013;61:4354-4364.

12. Malmgren S, Ciosek K, Lindblad R, et al. Electrochim Acta. 2013;105:8391.

13. Wang HY, Wang FM. J Power Sources. 2013;233:1-5.

14. Światowska J, Lair V, Pereira-Nabais C, et al. Appl Surf Sci. 2011;257:9110-9119. 